\title{
Understanding the Role of Device Level Interoperability in Promoting Health - Lessons Learned from the SmartPersonalHealth Project
}

\author{
V. Stroetmann', R. Thiel', K. A. Stroetmann', P. Wilson ${ }^{2}$, M. Romao ${ }^{3}$, M. Strubin ${ }^{4}$ \\ 'empirica GmbH \\ ${ }^{2}$ Cisco Systems Inc. \& Continua Health Alliance \\ ${ }^{3}$ Intel Corp \& Continua Health Alliance \\ ${ }^{4}$ New Ventures SPRL \& Continua Health Alliance
}

\begin{abstract}
Summary
Objectives: To summarize lessons learned from the European Commission (EC) co-funded project SmartPersonal Health, a project to promote a greater understanding of the value of interoperability among Personal Health Systems (PHS) and between them and other eltealth systems, in the landscape of continuity of care and across multi-cultural environments in Europe.

Methods: Key concepts in PHS interoperability, challenges, barriers and benefits were discussed with stakeholders (policy makers, regulators, procurers, healthcare providers, health professionals, patient representatives, industry, researchers) in three consultation workshops and a final conference. The results were synthesized in final report to the European Commission.

Results: The survey and analysis presented, which are designed to set the scene on the key requirements of device level interoperability within a context of using sensors, signals and imaging informatics in healthcare, set out key interoperability standards for PHS as provided for in the Continua Health Alliance Guidelines and explores further the need for wider organisational and regulatory aspects of interoperability.

Conclusion: Achieving interoperability of eHealth systems is a complex process involving various actors and challenges far beyond technical and standardisation issues. For harnessing the key benefits of PHS, any interoperability scenario needs to account for value-based business cases for all stakeholders involved. It must foresee to enable seamless and consistent data and information flows by integrating and mixing devices used by patients/consumers at home, for remote monitoring, for home hospitalisation and/or within the hospital.

Keywords

Personal health system, interoperability, medical devices, personal health record, eHealth
\end{abstract}

Yearb Med Inform 2011:87-91
Note: The authors are a subset of the partners of the SmartPersonalHealth project. The work reported below draws extensively on the work of this project, which was funded by the European Commission in its $7^{\text {th }}$ Framework Programme on Research (Support Action FP7-248419). Full details of the project are available at http:// sph.continuaalliance.org/. The analyses and conclusions derived reflect solely the views of their authors, the European Commission is not liable for any use that may be made of the information presented here.

\section{Background: Personal Health Systems}

The ageing of populations represents a considerable challenge to health, and particularly to social-care systems around the globe. As populations age the number of people requiring (longterm) care grows, while the number of those who deliver it shrinks. Furthermore, as populations age the nature of care becomes chronic rather than acute, and the best location of care may be the home rather than an institution. Yet most healthcare is still largely reactive, addressing disease as it presents, rather than focussing preventative interventions; and is still largely location centric, with care delivered in hospital or primary care facilities, rather than on the move or in the patient's home.

Key elements of providing more personalised and proactive healthcare which would allow certain patients to be actively engaged in managing their conditions at home, at work and at leisure, are telemedicine and telehealth solutions which may also be referred to as Personal Health Systems (PHS). A good definition of the concept to of PHS is offered by the European Commission co-funded project $\boldsymbol{P H S} 2020$ [1] which states that: Personal Health Systems assist in the seamless provision of quality controlled, and personalised health services to individuals regardless of location. The project lists the key elements of PHS as:

- Ambient and/or body devices (wearable, portable or implantable), which acquire, monitor and communicate physiological parameters and other health related context data of an individual (e.g., vital body signs, biochemical markers, activity, emotional and social state, environment);

- Intelligent processing of the acquired information and coupling of it with expert biomedical knowledge to derive important new insights about an individual's health status;

- Active feedback based on such new insights, either from health professionals or directly from the system to the individuals, assisting in diagnosis, treatment, rehabilitation and 
social care as well as in disease prevention and lifestyle management. A key player in the domain of promoting the use of PHS is the Continua Health Alliance [2], a non-profit, open industry coalition of healthcare and technology companies dedicated to establishing a system of interoperable personal health solutions to foster independence, empower individuals and provide the opportunity for truly personalised health and wellness management. Continua issues design guidelines which contain references to the standards and specifications that Continua selects for ensuring the interoperability of devices and advanced information technology to allow health data to be efficiently and safely shared to facilitate more personalised healthcare that can be delivered and supported where and when patients want it: at home, on the move, at work or at leisure [3].

Given its history in driving adoption of PHS and its wide range of member organisations, the Continua Health Alliance, together with partner organisations IHE - Integrating the Healthcare Enterprise [4] and ETSI - European Telecommunications Standards Institute [5] was awarded funding by the European Commission in 2010 to deliver a study named SmartPersonalHealth whose key objective was to raise the awareness and understanding of the concept and values of interoperability in PHS amongst key players.

The present survey, which is designed to set the scene on the key requirements of device level interoperability with in a context of using sensors, signals and imaging informatics in healthcare, is closely based on the work undertaken in the SmartPersonalHealth study.

a It is one of the various European Union activities following the publication by the Commission of the European Communities - COM (2008) 3282: Commission Recommendation on cross-border interoperability of electronic health record systems, Brussels, 2nd July 2008. See also [6].

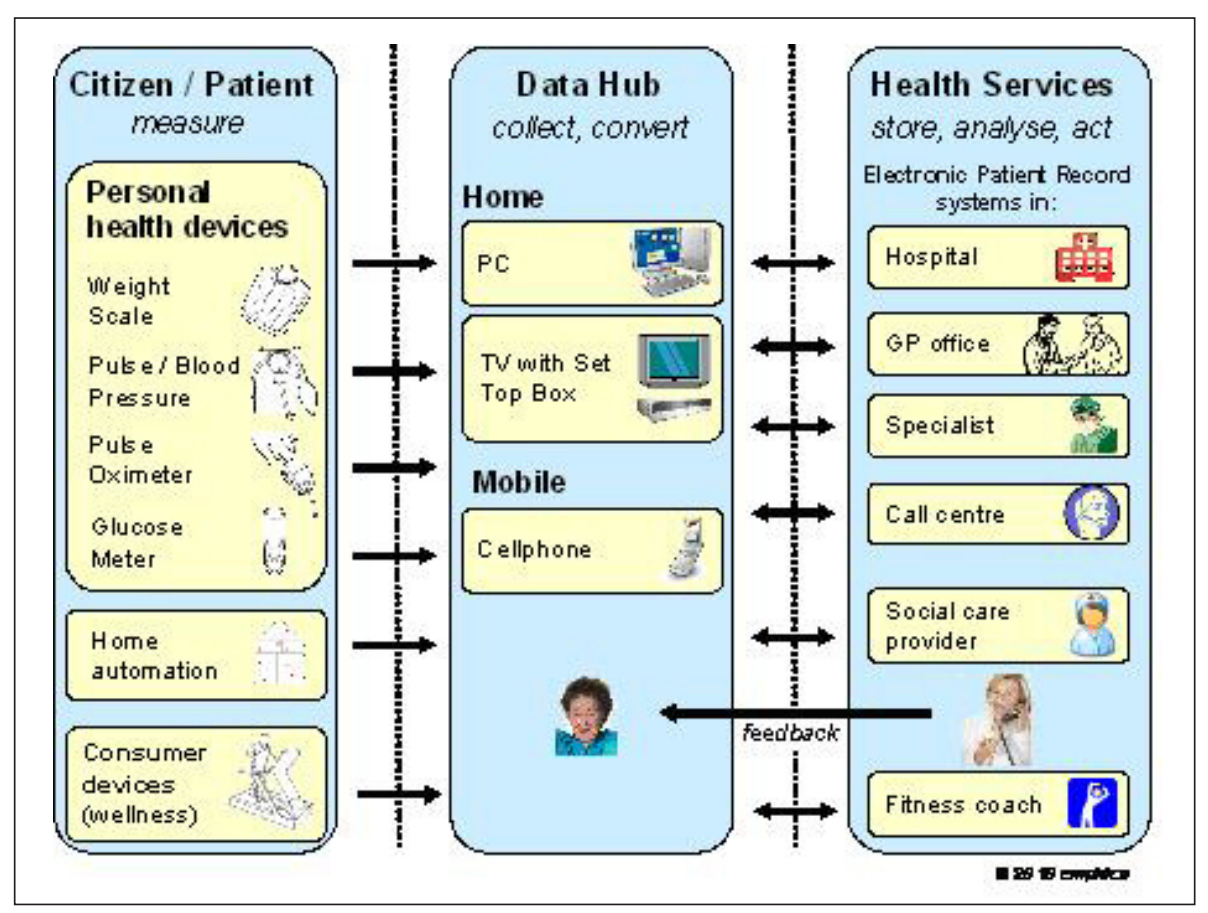

Fig. 1 Examples for data exchange in PHS-based health and care services

\section{The Ecosystem of Connected Health: Seamless Exchange of Data across PHSs}

In a generic scenario of PHS based solutions, patient data are transferred from personal devices through a data hub to a health service provider system, e.g. electronic patient record (EPR), electronic medical record (EMR), a hospital information system (HIS) or a General Practitioner patient system.

To give a more concrete sense of the technology behind the interoperability efforts which, ultimately, allow a seamless flow of data across a PHS system, consider the following use case of communicating remote monitoring information to EHR and PHR [7]. The patient uses the remote monitoring device to gather specific measurements. These are communicated either immediately or accumulated periodically (e.g., hourly, daily). Measurements could be communicated to a hub or device intermediary, or directly to the patient's PHR or clinician's EHR. "With appropriate safe- guards for patient privacy and security, a care coordinator ${ }^{\mathrm{b}}$ may review the measurement information" received via a portal or within an EHR. "Care coordinators may interact directly with the patient or caregivers to verify the information received and gather additional information about the patient's situation. If a clinician review, analysis, or intervention is needed, remote monitoring information and relevant additional information about the patient's situation is communicated to the clinician's EHR. The clinician reviews the remote monitoring information received and determines if a patient evaluation or change in treatment plan is necessary. Upon completion of the patient evaluation and modified treatment plan, the appropriate information may be communicated to the care coordinator and the patient's PHR." [8]

\footnotetext{
b In the U.S. context, care coordinators assist the patient and/or clinician in managing remote monitoring information and could include clinicians, nurses, caregivers, case managers, home health resources, and payor case managers.
} 
As can be deduced from Figure 1, even in a simple PHS scenario, a vast number of specific interoperability issues arise.

Interoperability between device and data hub: In order to support a wide range of diseases, it is necessary for such a PHS system to work with a large variety of measurement devices, such as blood pressure monitors, weighing scales, glucose meters, pulse oximeters, ECG monitors, peak flow meters, etc. For each of these measurement device types there are a number of manufacturers, but no one company manufactures all of these devices. So a telehealth system vendor will need to work with different suppliers to provide a complete set of measurement devices to its customers. Both the $\mathrm{HC}$ provider customer and the consumer or patient customer will have a strong interest in seamless and effortless interoperability between all such devices so that they can enjoy the freedom of buying whichever device best suits their needs, irrespective of the data hub or other devices that it will need to communicate with.

Interoperability between data hub and health service provider ICT application: A complementary need for cooperation emerges also at the interface of the hub transferring PHS data into electronic patient or medical records (EPR/EMR). Often the supplier of a PHS system is not the supplier of the EPR or EMR system that is used to store, integrate, analyse and display health data about the patient. Again here the customer simply wants systems to interoperate and does want to be tied into one particular system.

The freedom that the patients and healthcare providers want can only be delivered if the vendors can insure interoperability by use of standards and adherence to use case guidelines.

\subsection{Standards for Data Collection, Aggregation and Processing}

The Continua Guidelines [9] seek to support the use of such recognised

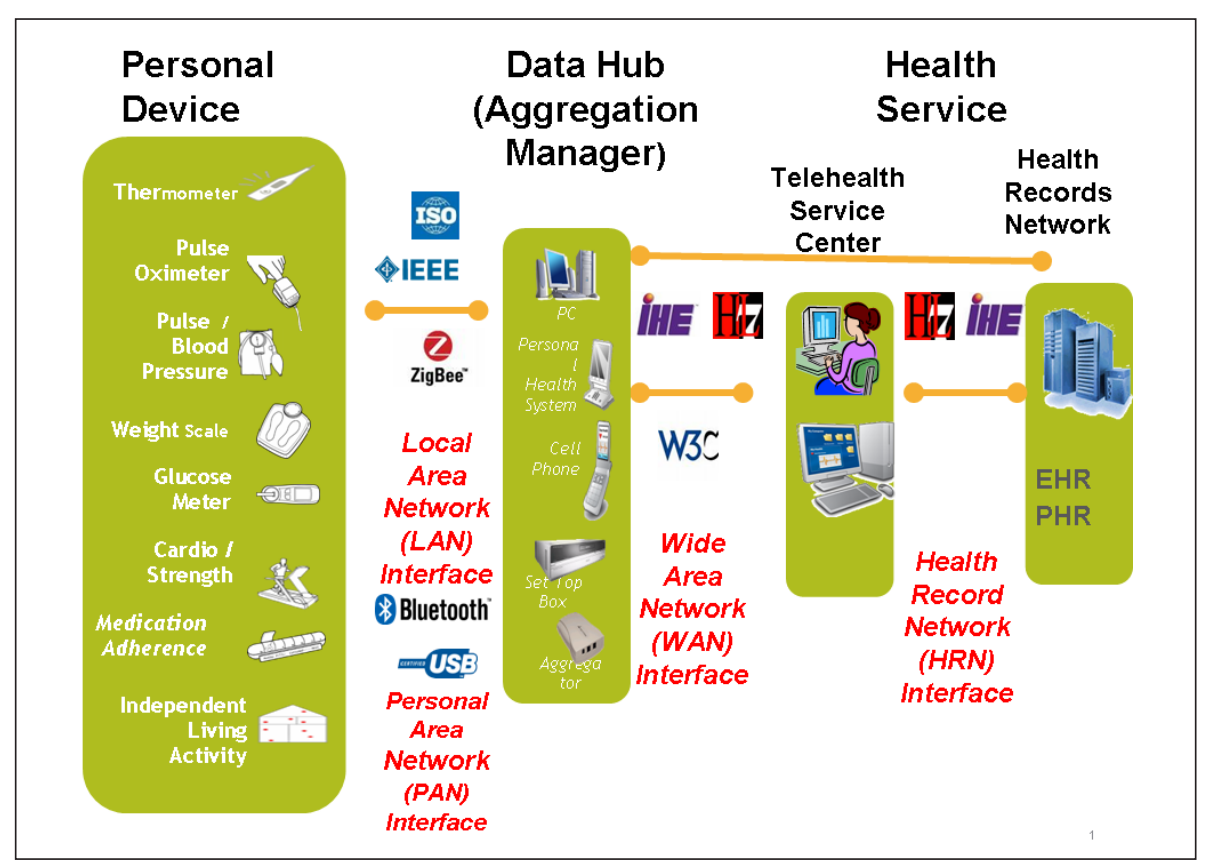

Fig. 2 Overview of interface standards. Source: Continua Health Alliance

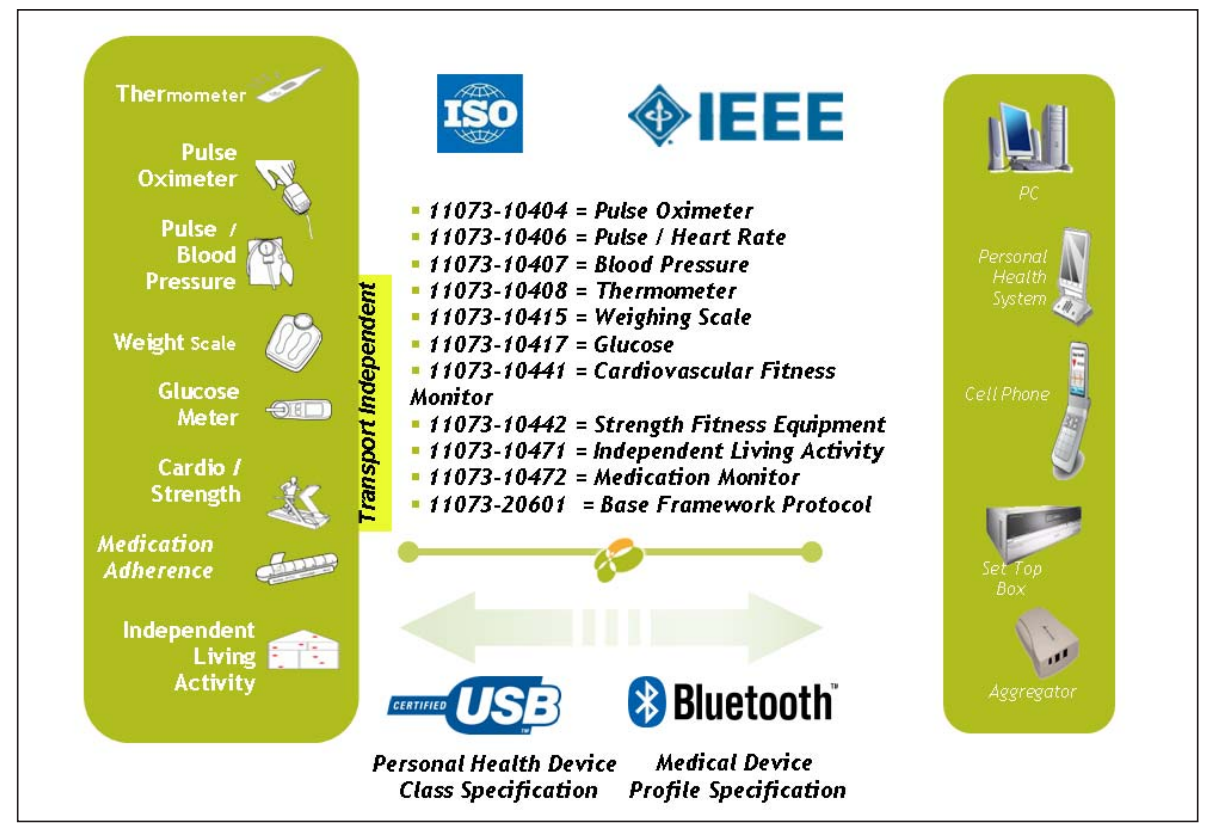

Fig. 3 Continua device connectivity standards. Source: Continua Health Alliance

standards by creating a number of use cases and detailing how each standard is to be applied in order to allow a seamless flow of data across a PHS system. Figure 2 presents an overview of the selected interface standards necessary in typical PHS scenario.

The actors in this interoperability paradigm - based on communication needs are: the personal area network 
devices (PAN) for the measurement exchange around a person; local area networking devices (LAN) for the measurement exchange at a location, application hosting devices (AHD) such as personal computer, cell phone, etc.; wide area networking device (WAN); and a health record/reporting application(HR). The interfaces between these network devices are defined as the PAN, LAN, WAN, and health record network (HRN) interfaces.

The interface is further structured into three distinct layers, with appropriate standards selected to represent the individual layers and establish interoperability in the Personal Health ecosystem. The three PAN layers are transport, optimised data exchange protocol and device specialisation layer. Continua has constrained the lower level protocol standards for communication to USB and Bluetooth. For the optimized exchange of information in the PAN Interface Continua has selected the ISO/IEEE Std 11073-20601 version 1.0 Personal Health Device Communication. The internationally harmonized standard has definitions and structures in place to convert from an abstract data format into a transmission format.

The ISO/IEEE Std 11073-20601 version 1.0 protocol acts as a bridge between device specific information captured in individual device specializations and the underlying transports to provide a framework for optimized exchange of interoperable data units across the PAN interface. The device specialisations are listed in Figure 3.

Similarly standards are identified on the LAN interface (e.g., ZigBee) WAN interface (HL7 and IHE) and HRN interfaces so that patient information can be sent safely from a WAN device (sender) to an electronic health record device (receiver). The WAN device could be the Telehealth Centre/Remote Patient Monitoring server of a Disease Management service provider or the Application Server of a Social Care, Ageing Independently or Health \& Fitness service provider. The patient information for transfer may include a report summarising the patient's current status, a detailed listing of specific patient results, readings from one or more personal health devices, or a combination of these. The electronic health record device may contain a hospital's Enterprise Health Record (EHR), a physician's Electronic Medical Record (EMR) or a Personal Health Record service (PHR) used by the patient.

To facilitate the accurate transfer of both coded patient results from personal health devices and textual summary results from patient care-givers, the HL7 Personal Healthcare Monitoring Report document format standard was chosen. This standard is close to the widely used Continuity of Care Document (CCD) standard with specific changes to accommodate device data monitoring.

A future is envisioned where patient information is sent between providers by various methods. These methods include: secure direct connection over the Internet, secure email, delivery on portable media (data stick, etc.), through a messaging hub, and through a data repository or Regional Health Information Organisation or a National Health Information Network. To facilitate this, a messaging standard supported by IHE that certify electronic health record systems - IHE's CrossEnterprise Document Reliable Interchange (XDR) profile - was chosen. This profile is based on the XDS.b (Cross-Enterprise Document Sharingb) profile and the underlying standards that support it (SOAP 1.2, ebXML 3.0, MTOM, etc.).

Finally, to accomplish secure direct communication of pertinent patient information between care-givers, the IHE XDR (Cross-Enterprise Document Reliable interchange) profile utilizes current standards such as SOAP 1.2 and MTOM.

The IHE Patient Care Device domain (PCD), formed in 2005, addresses the integration of medical devices into the healthcare enterprise, potentially resulting in significant improvements in patient safety and quality of care. IHE aligns well with Continua's vision of profiling existing standards and constraining them for interoperability. The PCD domain has built a technical framework of use cases which have defined profiles describing transactions (with interfaces) and actors. Each of the profiles represents an interface in which the actors are defined and a standard or standards identified for that specific interface and/or transaction. Relevant profiles include the PCD-01, the Alarm Communication Management (ACM) profile, and the Rosetta Terminology Mapping (RTM) profile with the development of a "Rosetta Stone" that correlates each vendors' internal terms and units of measure for each of the IEEE 11073 defined reference identifications.

\section{Key Challenges for Realising full PHS Interoperability}

The Continua Guidelines implementing IEEE and ISO standards will allow a component manufacturer to identify with which interface standards he must comply in order to allow for a seamless flow of data. If the appropriate standards are correctly implemented, the device would then comply with Continua Guidelines and would thus be eligible for certification, which will in turn assure the purchaser (who may be an individual consumer or a public procurer) that the various components purchased will interoperate and that the PHS system can be established. The Continua Guidelines and the standards to which they refer will therefore go some considerable way towards driving device level interoperability.

Because of the lack of standards in PHR specification [10], and given the diversity in implementing PHRs in real context, the sharing of patient information between health service providers and across the medical specialities poses a host of technical challenges. A complementary need for cooperation emerges at the 
interface of the hub transferring personal telehealth data into electronic patient or medical records (EPR/EMR). Often the supplier of a telehealth system is not the supplier of the EPR or EMR system that is used to store, integrate, analyse and display health data about the patient. Since there were no proper standards in place yet to transfer health data from a telehealth system into such a health service provider system, the telehealth vendor had to work with all major EPR or EMR system providers to develop custom interfaces for transferring this data. A large amount of work remains to overcome this significant barrier for proper integration of telehealth data into other systems and thus limiting the potential health benefits and efficiency improvements that personal telehealth could offer.

However, a functional PHS is not achieved solely through device level interoperability. Achieving interoperability of eHealth systems is a complex process involving various actors and challenges far beyond technical and standardisation issues. Interoperability of eHealth systems, defined in the broader context of health system interoperability, is the ability, facilitated by ICT applications and systems, to exchange, understand and act on citizens/patients and other health-related information/knowledge among organisationally, linguistically and/or culturally disparate health professionals, patients and other actors and organisations, within and across health system jurisdictions and administrations in a collaborative manner [11].

In the clinical practice, interoperability issues to be tackled span a wide range of organisational, legal and semantic challenges. At the organisational level, for instance, the integration of PHS into clinical workflow implies also organising processes for reaction, acting on data and signals from PHS such as alerts in cases of, e.g., a decomposition of a patient or other emergencies. This means that detailed guidelines need to be prepared for action and follow up, protocols for physicians, nurses or other health professionals as well as call centres, patients and carers need to be designed and all actors involved must be well informed and trained. Integrated workflow processes need to be studied in detail, understood and optimally supported by the ICT solution.

Seamless exchange of patient data and full integration of PHS into daily routine presume an underlying ICT infrastructure with regulated access, identity management, authentication mechanisms, clear security policy, audit trail and further rules. Such an infrastructure would enable also consistent and transparent data collection and analysis for, e.g., outcome measurements which can eventually provide the basis for outcome oriented reimbursement. Semantic issues need to be addressed in relation to a specific domain, for example diabetes management, in languages to be understood both by professionals and by lay persons. Capabilities of integrated PHS-PHR/EHR solutions for providing decision support or data analysis for population health may need to be considered. Ultimately, different types of health professionals may have different needs and requirements to design and integration, and also different "business" interests.

In sum, for harnessing the key benefits of PHS, any interoperability scenario needs to account for value-based business cases for all stakeholders involved. It must foresee to enable seamless and consistent data and information flows by integrating and mixing devices used by patients/consumers at home, for remote monitoring, for home hospitalisation and/or within the hospital. Such continuous exchange of data can only be realised once an organisational, legal and technical framework has been developed, and furthermore a process has been initialised to interconnect systems and actors, and that allows agreements for adopting common standards.
Further support for faster advancing eHealth systems interoperability can also be expected from the 2010 Digital Agenda for Europe. It underlines "the right of individuals to have their personal health information safely stored within a healthcare system accessible online" as an essential condition for successful uptake of eHealth and calls for actions to remove legal and organisational barriers, particularly those to pan-European interoperability [12].

\section{References}

1. http://ec.europa.eu/information_society/activities/ health/docs/projects/phs2020/phs2020-bookrev16082009.pdf.

2. www.continuaalliance.org.

3. Piniewski B, Muskens J, Estevez L, Carroll R, Cnossen R. Empowering Healthcare Patients with Smart Technology. Computer 2010;43(7):27-34. doi:10.1109/MC.2010.200

4. www.ihe.net

5. www.etsi.org

6. Lewalle P, Rodrigues JM, Zanstra P, Ustun B, Kalra D, Surian G, et al. A Deployment and Research Roadmap for Semantic Interoperability: the EU SemanticHEALTH project. Stud Health Technol Inform2008;136:635-40.

7. Adapted from, Remote Monitoring Detailed Use Case', U.S. Department of Health and Human Services, Office of the National Coordinator for Health Information Technology; 2008.

8. See reference no. 7, p. 18.

9. Continua Version One Design Guidelines, 2009, http://www.continuaalliance.org/products/designguidelines.html. In 2010, an extended update of version one Design Guidelines was published (v1.5)

10. ISO/HL7 NP 16527 Personal Health Record System Functional Model, Release 1 (PHRS FM), is still under development; http://www.iso.org/iso/ iso_catalogue/catalogue_tc/catalogue_detail. htm? csnumber $=57046$

11. Stroetmann VN, editor; Kalra D, Lewalle P, Rodrigues JM, Stroetmann KA, Surjan G, Ustun B, et al. Semantic Interoperability for Better Health and Safer Healthcare: Research and Development Roadmap for Europe: SemanticHEALTH Report. European Commission; Luxembourg: Office for Official Publications of the European Communities; 2009.

12. European Commission: A Digital Agenda for Europe. Brussels, 19.05.2010 - COM(2010)245. See esp. sub-objective 7.2: "Sustainable healthcare and ICTbased support for dignified and independent living".

Correspondence to:

Petra Wilson

Tel: + +32474495999

E-mail: petrwils@cisco.com 